Cultural Transmission and the Evolution of Cooperative Behavior

Author(s): Robert Boyd and Peter J. Richerson

Source: Human Ecology, Vol. 10, No. 3, Biology and Culture (Sep., 1982), pp. 325-351

Published by: Springer

Stable URL: http://www.jstor.org/stable/4602658

Accessed: 11/01/2011 07:24

Your use of the JSTOR archive indicates your acceptance of JSTOR's Terms and Conditions of Use, available at http://www.jstor.org/page/info/about/policies/terms.jsp. JSTOR's Terms and Conditions of Use provides, in part, that unless you have obtained prior permission, you may not download an entire issue of a journal or multiple copies of articles, and you may use content in the JSTOR archive only for your personal, non-commercial use.

Please contact the publisher regarding any further use of this work. Publisher contact information may be obtained at http://www.jstor.org/action/showPublisher?publisherCode=springer.

Each copy of any part of a JSTOR transmission must contain the same copyright notice that appears on the screen or printed page of such transmission.

JSTOR is a not-for-profit service that helps scholars, researchers, and students discover, use, and build upon a wide range of content in a trusted digital archive. We use information technology and tools to increase productivity and facilitate new forms of scholarship. For more information about JSTOR, please contact support@jstor.org. 


\title{
Cultural Transmission and the Evolution of Cooperative Behavior
}

\author{
Robert Boyd $^{1}$ and Peter J. Richerson ${ }^{2}$
}

Sociobiological theory predicts that humans should not cooperate with large groups of unrelated individuals. This prediction is based on genetic models that show that selection acting on variation between large unrelated groups will generally be much weaker than selection acting on variation between individuals. Recently, several authors have presented related models of human evolution that integrate cultural and genetic transmission of behavior. We show that in such models group selection is potentially a strong force. Data on ethnocentrism is examined in the context of these results.

KEY WORDS: altruism; cultural inheritance; dual inheritance; group selection.

\section{INTRODUCTION}

Sociobiology predicts that human behavior should be fundamentally egoistic. Natural selection, it is argued, has resulted in human beings who seek increased access to resources, greater personal security, and all those things that lead (or at least led during some earlier age) to greater reproductive success. This view of human nature is certainly not unique to sociobiology. In the social sciences, it goes back at least to Hobbes and Hume, and it is the fundamental paradigm of modern Western economic theory. Within the social sciences, however, human egoism is a postulate which is confirmed to the extent that the theories built upon it accurately reflect reality. Within sociobiology, it is not a postulate but a conclusion based on deductions from evolutionary theory.

${ }^{1}$ School of Forestry and Environmental Studies, Duke University, Durham, North Carolina 27706.

${ }^{2}$ Division of Environmental Studies, University of California, Davis, California 95616. 
Compared with most other species of organisms, humans cooperate on a very large scale. In commerce, warfare, and politics, large numbers of individuals act in concert to achieve common goals. The scale of human cooperation is difficult to explain in terms of choice by self-interested actors. Cooperation commonly entails the production of what economists term "public goods." A public good is a commodity or service which, having been produced by one member of the group, cannot feasibly be withheld from other members (Olson, 1971). Modern examples of public goods include improved air quality or a better union contract. There is an extensive literature in economics dealing with the voluntary production of public goods (e.g., Olson, 1971; Buchanan, 1968; Taylor, 1976), which concludes that individuals will not cooperate in large groups to provide themselves with public goods in the absence of coercion.

The simplest way to explain the scale of human cooperation is to assume that humans are not solely self-interested-that in making choices individuals balance changes in their own welfare against changes in the welfare of others. Examples of such noneconomic paradigms in the social sciences include substantive economics in anthropology and functionalism in sociology. Such views of human nature are subject to two related objections. First, the evolution of such "altruistic" humans would require rather special circumstances. Sociobiological theory holds that natural selection should favor cooperation only among genetically related individuals. In special circumstances, it is thought that this process, called "kin selection," can lead to extensive cooperation, as for example among the social insects. However, for the ecological and demographic parameters that characterize most vertebrates, cooperation will be limited to small groups of related individuals. With the exception of humans, this result is consistent with the data. Second, in most societies, individuals belong to more than one social grouping, for example, a clan, an ethnic group, a social class, or a state. The interests of these groups often conflict with each other and with the individual's own interests. In making choices, how much weight should individuals give their own interests and the interests of each of the groups to which they belong? For sociobiologists, the theory of kin selection gives relatively clear answer to these questions. Alternative theories explaining the extensive non-kin cooperation in the human species need to be similarly specific.

In this paper we present a model that can overcome these two problems. The model assumes that humans inherit their social behavior culturally. Recently, several authors have presented models of cultural inheritance (Campbell, 1965, 1975; Durham, 1976; Cavalli-Sforza and Feldman, 1981; Richerson and Boyd, 1978; Boyd and Richerson, 1980; Pulliam and Dunford, 1980; Lumsden and Wilson, 1981) that attempt to explain macroscopic patterns of cultural variation in terms of everyday 
events in the lives of individuals. In these models, patterns of enculturation and social interaction produce "forces" that affect the frequency of different culturally transmitted variants. For example, culturally transmitted variation between groups could lead to a "group selection" process that favors cultural variants that enhance group success. Campbell (1975) argued that the counter-hedonic nature of most systems of moral teaching suggests that these cultural traits have been shaped by such a group selection process. The models presented in this paper formalize and extend Campbell's reasoning. Because the rules of cultural transmission are often different from the rules of genetic transmission, the patterns of variation that result from cultural transmission may also be quite different. We argue that in some circumstances this can cause group selection to be a strong force favoring traits that lead to cooperation among large groups of genetically unrelated individuals, and that these models are consistent with the origin of the human capacity for culture via ordinary Darwinian processes. We conclude by considering the cross-cultural evidence about ethnocentrism in the light of our model.

\section{COOPERATION AND PUBLIC GOODS}

Throughout this̀ paper we assume that social cooperation entails the production of public goods, and that in large groups rational selfish individuals will not voluntarily cooperate to produce public goods. There is a substantial literature dealing with this topic, and it is only necessary to sketch the basic reasoning here. A rational self-interested individual is one who seeks to maximize a utility function that is a function only of his or her own consumption of various goods, i.e., he or she is indifferent to changes in the consumption of others. A public good is a good that, once it is produced by one member of the group, cannot be feasibly withheld from other members. Many of the fruits of social cooperation are public goods. The modern state provides law and order within its boundaries and protection from attack by other countries; labor unions provide higher wages and cartels higher prices. In each case, large groups of unrelated individuals cooperate to pursue a common goal. The achievement of such a goal leads to benefits that flow to all members of the group without regard to their contribution. Most economists believe that, except in very special circumstances, rational selfish individuals will not voluntarily invest in the provision of public goods for large groups (Olson, 1971; Buchanan, 1968; for a contrary view, see Taylor, 1976). Because of the collective nature of public goods, the incremental benefit to an individual that results from his or her own investment of time, effort, or resources in the production of a public good is usually quite small. On the other hand, the incremental cost of this 
investment to his or her own well-being is usually substantial. When the incremental cost exceeds the incremental benefit, a rational individual does not invest.

It might be argued that individuals cooperate in order to avoid punishment by other members of their own group. This notion seems plausible based on common experience. However, it does not solve the theoretical problem; it only raises the new problem of why individuals should cooperate to punish other individuals. Punishment itself is an investment in the production of some other public good, for example, civil order. Each potential punisher can have only a small incremental effect on the level of civil order, and again, the cost to the individual participating in the punishment of others could be substantial. The rational selfish individual would let the other person do the punishing.

It may be that repeated interactions among a finite group of rational selfish individuals may lead to voluntary cooperation via reciprocity. Theoretical studies (Shubik, 1971; Taylor, 1976; Axelrod, 1980a, 1980b; Axelrod and Hamilton, 1981) indicate that in groups of two, reciprocity can have a strong effect. In larger groups it seems much less likely (Taylor, 1976; Kurz, 1977). Given that humans often cooperate in very large groups, it would seem useful to pursue other possible explanations.

\section{CULTURAL INHERITANCE}

Cultural inheritance occurs whenever individuals acquire determinants of their behavior from other individuals via modeling, imitation, or some related process. By acquire, we mean that in order to predict the behavior that characterizes a particular individual (call him or her ego), one must know the behaviors that characterize the set of individuals (we will call them "cultural parents") from whom ego acquired his or her behavior. The set of cultural parents can be drawn from the previous generation or from among ego's contemporaries. Following Cavalli-Sforza and Feldman (1981), we refer to the first case as vertical (from the biological parents) or oblique (from others of the parental generation) transmission, and the second as horizontal transmission. Clearly, the acquisition of any culturally transmitted traits entails vertical, oblique, and horizontal transmission.

It follows from this definition of cultural inheritance that the distribution of behaviors that characterizes a human population depends on the distribution of behaviors in the same population during previous time periods. To predict the equilibrium distribution of culturally acquired behaviors in the population, one must consider the forces that act to change the distribution of those behaviors through time. Among these are forces analogous to those that affect the distribution of genotypes in a population. 
Errors in cultural transmission analogous to genetic mutuation presumably occur. Similarly, in finite populations sampling errors must lead to the cultural analog of genetic drift. Finally, individuals with different culturally acquired behaviors must often have different probabilities of achieving the necessary social position so that they themselves may be imitated. This creates the cultural analog of natural selection. We have argued elsewhere (Richerson and Boyd, 1978; Boyd and Richerson, 1980, 1981) that natural selection acting on culturally acquired variation will often favor traits that are genetically disadvantageous.

There are also forces that act on the distribution of the culturally transmitted trait that do not have analogs in the usual models of population genetics. These forces arise for two reasons. First, traits acquired via de novo learning can be culturally transmitted, leading to a "Lamarckian" force caused by the transmission of guided variation. Second, the rules of cultural transmission can affect the distribution of culturally transmitted traits if some traits are more likely to be imitated than others. We call this general category of forces biased transmission. There is a growing theoretical literature that analyzes the interaction of these forces (for references, see Cavalli-Sforza and Feldman, 1981; Boyd and Richerson, 1980; and Lumsden and Wilson, 1981).

Here we analyze models that include a particular kind of transmission bias that we have labeled "conformist transmission." Individuals are assumed to be disproportionately likely to imitate the more common behavioral types among their cultural parents. This creates a force that increases the frequency of more common types in the population. In this section we analyze how this force affects the dynamics of different behavioral variants in a population. In the next two sections, we show how conformist transmission might act to enhance genetic fitness, and then argue that conformist transmission can cause group selection to be a strong force in determining the frequency of different culturally transmitted behaviors.

To see how conformist transmission acts to produce a force affecting the frequency of traits in a population, consider the very simple case of vertical transmission of a dichotomous trait by sets of three cultural parents. Each individual can be characterized by one of two behaviors, labeled $\mathrm{C}$ and $\mathrm{D}$. The probability that an individual acquires trait $\mathrm{C}$, given that he or she is exposed to a particular set of parents, is shown in Table I. This cultural transmission rule is particularly simple because each of the parents is assumed to have the same role. It does not matter which parents are $\mathrm{C}$, only how many are $\mathrm{C}$. Let $q_{t}$ be the frequency (i.e., the fraction) of $\mathrm{C}$ individuals in the population during generation $t$. Let $M\left(y \mid q_{t}\right)$ be the probability that a set of cultural parents has $y(y=1,2,3)$ individuals who are iype $\mathrm{C}$, given that the frequency of $\mathrm{C}$ in the population is $q_{t}$. Let $T(y)$ be the probability that an individual exposed to such a set of cultural parents acquires the trait $\mathrm{C}$. The frequency of individuals characterized by $\mathrm{C}$ in the 
Table I. Probability That Each Possible Combination of Three Cultural Parents Results in an Offspring Who Is Variant $C^{a}$

\begin{tabular}{cccc}
\hline Parent 1 & Parent 2 & Parent 3 & $\begin{array}{c}\text { Probability that offspring } \\
\text { acquires variant C }\end{array}$ \\
\hline C & C & C & 1 \\
C & C & D & $2 / 3+A / 3$ \\
C & D & C & \\
D & C & C & $1 / 3-A / 3$ \\
D & D & C & \\
D & C & D & 0 \\
C & D & D & \\
D & D & D & \\
\hline
\end{tabular}

${ }^{a}$ The probability that the offspring acquires variant $\mathrm{D}$ is one minus the probability that it acquires $\mathrm{C}$.

population after cultural transmission is $q^{\prime}{ }_{t}$, which is given by:

$$
q_{t}^{\prime}=\sum_{y=1}^{3} M\left(y \mid q_{t}\right) T(y)
$$

Equation (1) says: to compute the proportion of type $\mathrm{C}$ individuals after transmission, one multiplies the probability that an offspring acquires trait $\mathrm{C}$, given that he or she is exposed to $y$ cultural parents with trait $\mathrm{C}$, and then sums over all possible values of $y$.

If one assumes that sets of cultural parents are a random sample of the population, then (1) can be simplified to ${ }^{3}$

$$
q_{t}^{\prime}=q_{t}+A q_{t}\left(1-q_{t}\right)\left(2 q_{t}-1\right)
$$

There are several things to note about Eq. (2). First, if $A=0$, cultural transmission leaves the frequency of traits unchanged. We will refer to this as a linear transmission rule. Any deviation from linearity in transmission creates forces that affect the frequency of culturally transmitted traits. Second, if $A>0$, cultural transmission creates a force increasing the frequency of the more common variant in the population. That is, $q_{t}>0.5$ causes $q^{\prime}{ }_{t}>q$ and $q_{t}<0.5$ causes the reverse. The parameter $A$ gives the

${ }^{3}$ With random mating

$$
M\left(y \mid q_{t}\right)=\left(\begin{array}{l}
3 \\
y
\end{array}\right) q_{t}\left(1-q_{t}\right)^{3-y}
$$

and noting that $T(3)=1=3 / 3$ it follows that Eq. (1) can be rewritten as

$$
\begin{aligned}
\mathrm{q}^{\prime} & =(1 / 3) \sum_{y=1}^{3}\left(\begin{array}{c}
3 \\
\dot{y}
\end{array}\right) y q_{t}^{y}\left(1-q_{t}\right)^{3-y}+A\left[q_{t}^{2}\left(1-q_{t}\right)-\left(1-q_{t}\right)^{2} q_{t}\right] \\
& =q_{t}+A q_{t}\left(1-q_{t}\right)\left(2 q_{t}-1\right)
\end{aligned}
$$


deviation of the cultural transmission rule from linearity. Finally, if $A<0$, the rare trait in the population is increased.

This simple result can be extended in a variety of ways. It is possible to show that allowing different cultural parent to have different linear weightings yields the same result, and that the result can be generalized to any number of cultural parents greater than 2. Similar results can be obtained for horizontal transmission by supposing that a particular individual encounters $m-1$ other individuals, and then, given his own phenotype and that of the others, acquires (or remains) type $\mathrm{C}$ with a given probability. The results for this model are identical to a vertical model in which parents have different weightings, except that the generation time is now the time it takes to encounter $m-1$ other individuals. The model can also be extended to include the nonrandom formation of sets of cultural parents. Linear assortative formation of sets of cultural parents weakens the force due to the nonlinear transmission rule. Finally, the model can be generalized to allow more than two variants of the cultural trait. Again, the transmission rule always decreases the frequency of the least common trait. These results indicate that the general result given in Eq. (2) is fairly robust.

\section{THE ADAPTIVE ADVANTAGES OF CONFORMIST TRANSMISSION}

We have argued that conformist transmission has important consequences for the evolution of social behavior. Clearly, it would be of interest to know if there are any circumstances in which individuals who acquire their behavior via conformist cultural transmission will, on average, have higher fitness than individuals who utilize a cultural transmission rule without the conformist effect. One such situation may exist when a population is subdivided into many subpopulations in which different behaviors are favored by selection. In such subdivided populations migration between subpopulations will often maintain a polymorphism in each subpopulation. In many cases, however, the behavioral variant favored by selection in a particular subpopulation will be the most common variant. Under these conditions, conformist transmission is a simple general rule that increases the probability that an individual will acquire the locally favored variant.

A complete analysis of this argument is beyond the scope of this paper. The following simple model will illustrate how conformist transmission may be adaptive in at least some kinds of subdivided populations, and will also serve to introduce the life cycle and population structure that we use in the next section. Suppose that a very large population of individuals is subdivided into $n$ subpopulations, or "demes." Each individual is characterized by one of two behaviors, labeled C and D. For simplicity we will assume that this trait is acquired via some combination of vertical and oblique cultural transmission from three 
cultural parents, and that the members of the set of cultural parents for each naive offspring are drawn randomly from the individuals present in each subpopulation. As indicated above, we believe that this argument will apply to much more general models. The subpopulations are assumed to be large enough that any variation between subpopulations due to sampling error can be ignored.

The "life cycle" of cultural transmission is diagrammed in Fig. 1. To simplify the analysis, we have divided this into discrete nonoverlapping stages. The frequency of $\mathrm{C}$ individuals among parents just before cultural transmission in the $i$ th subpopulation during generation $t$ is labeled $q_{i, t}$. After transmission, the frequency of $\mathrm{C}$ individuals in the $i$ th subpopulation during generation $q_{i, t}$ will be different from $q_{i, t}$ if cultural transmission is nonlinear.

We will assume that $q_{i, t}^{\prime}$ is related to $q_{i, t}$ as follows:

$$
q_{i, t}^{\prime}=q_{i, t}+A q_{i, t}\left(1-q_{i, t}\right)\left(2 q_{i, t}-1\right)+B_{i} q_{i, t}\left(1-q_{i, t}\right)
$$

The second term on the right side of (3) results from conformist transmission by three or four parents, favoring the more common variant in the population. The third term represents a directional force that favors one variant or the other at all frequencies of $q_{i, t}$. If $B_{i}$ is positive, this force increases the frequency of $C$ individuals in the $i$ th subpopulation; if it is negative, the opposite occurs. This force occurs if offspring are predisposed to adopt one variant. The predisposition could be inherited either genetically or during an earlier episode of cultural transmission. We will refer to this as "direct bias." Cultural transmission rules with direct bias have been extensively analyzed by Cavali-Sforza and Feldman (1981).

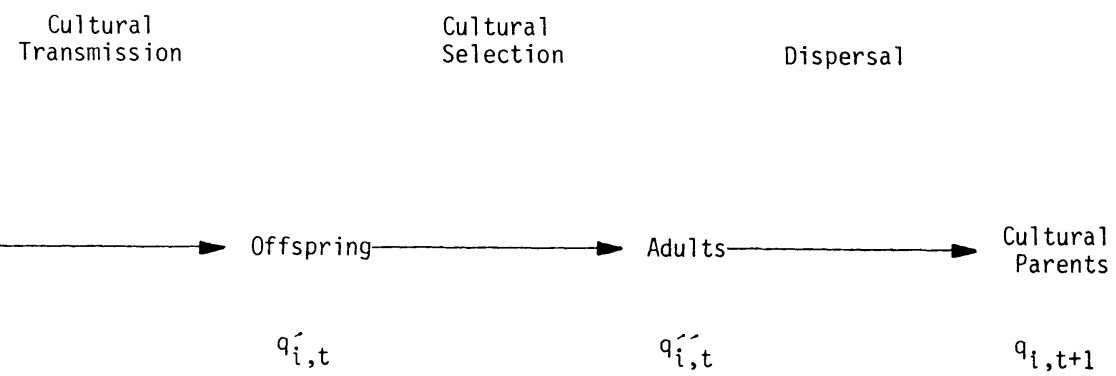

Fig. 1. This figure diagrams the assumed life cycle in the model of cultural selection. The frequency of $\mathrm{C}$ individuals in the $i$ th subpopulation at each stage in the life cycle is labeled $q_{i, t}, q_{i, t}^{\prime}$, etc. 
If individuals are to socialize offspring during the next generation they must survive and acquire whatever resources or social positions are necessary for cultural parenthood. Let $V_{i, C}$ and $V_{i, D}$ be, respectively, the probability that individuals characterized by behavior $C$ and $D$ enter the pool of potential cultural parents in the $i$ th subpopulation. Then the frequency of $\mathrm{C}$ individuals in the $i$ th subpopulation after selection is

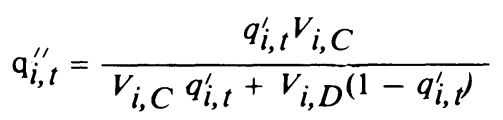

If the change in the frequency of $\mathrm{C}$ individuals during one generation is small, then (4) can be rewritten as follows: ${ }^{4}$

$$
q_{i, t}^{\prime \prime}=q_{i, t}+q_{i, t}\left(1-q_{i, t}\right)\left[U_{i}+A\left(2 q_{i, t}-1\right)\right]
$$

where

$$
U_{i}=B_{i}+\left(V_{i, C^{\prime}} V_{\mathrm{i}, \mathrm{D}}\right)-1
$$

The parameter $U_{i}$ gives the net directional effect of direct bias $\left(B_{i}\right)$ and selection $\left(V_{i, C} / V_{i, D}-1\right)$. Several authors (Durham, 1976; Alexander, 1979; Irons, 1979; Lumsden and Wilson, 1981) have argued that natural selection will shape individual predispositions so that direct bias will act to increase the frequency of the variant favored by selection. In the context of the present model, this would mean that $B_{i}$ and $\left(V_{i, C} / V_{i, D}-1\right)$ would have the same sign. There are at least two reasons, however, why this may not be true in every case. First, it may not be possible to create biases that favor the appropriate behavior in a variety of different habitats. Direct bias may often increase the frequency of adaptive behaviors on average, but decrease

${ }^{4}$ Note that (4) can be rewritten in the following form:

$$
q_{i, t}^{\prime \prime}=q_{i, t}^{\prime}(1+s) / 1+s q_{i, t}^{\prime}
$$

where $s=\left(V_{i, c} / V_{i, D}\right)-1$. Thus, if selection is weak, $s$ is small, and we can approximate the denominator by the first two terms in a Taylor series. This results in the following expression for $q_{i, t}^{\prime \prime}$

$$
q_{i, t}^{\prime \prime} \approx q_{i, t}^{\prime}+s q_{i, t}^{\prime}\left(1-q_{i, t}^{\prime}\right)
$$

Substituting the expression for $q_{i, t}^{\prime}$ into this equation results in Eq. (5) if terms of order $s B_{p} A B_{i}$ and $s A$ are ignored. 
them in particular habitats. Second, when environments are changing, the cultural transmission system may increase the frequency of variants favored by selection in previous environments but not in current ones. Here, however, we will only assume that the net effect of direct bias and selection within subpopulations on culturally transmitted variation is to increase the behavior with the higher genetic fitness.

The final stage of the life cycle is dispersal. Each individual has a probability $(1-m)$ of remaining in his or her natal subpopulation to reproduce, and a probability $m$ of emigrating. The individuals who emigrate have an equal probability of settling in each of the $n$ subpopulations, including the one they just left. Some density-dependent process regulates the size of each subpopulation so that each subpopulation contributes an equal number of migrants in each generation. Given these assumptions, the frequency of $\mathrm{C}$ individuals in the $i$ th subpopulation after migration, but before transmission, is

$$
q_{i, t+1}=(1-m) q_{i, t}^{\prime \prime}+(m / n) \sum_{j=1}^{n} q_{j, t}^{\prime \prime}
$$

for $i=1, \ldots, n$.

Using Eq. (7), one can show that the equilibrium frequency of $\mathrm{C}$ individuals in the $i$ th subpopulation, $\hat{q}_{i}$, must satisfy the following condition: ${ }^{5}$

$$
Q=\hat{q}_{i}-\frac{(1-m)}{m} \hat{q}_{i}\left(1-\hat{q}_{i}\right)\left(U_{i}+A\left(2 \hat{q}_{i}-1\right)\right)
$$

${ }^{5}$ For convenience let

$$
q^{\prime \prime} i, t=q_{i, t}+F\left(q_{i, t}\right)
$$

where the function $F\left(q_{i, t}\right)$ is defined by Eq. (5). Then from Eq. (7),

$$
q_{i, t+1}=\left[q_{i, t}+F\left(q_{i, t}\right)\right](1-m)+(m / n) \sum_{j=1}^{n}\left[q_{j, t}+F\left(q_{j, t}\right)\right]
$$

for $i=1, \ldots, n$. Thus at equilibrium,

$$
0=-m \hat{q}_{i}+F\left(\hat{q}_{i}\right)(1-m)+(m / n) \sum_{j=1}^{n}\left[\hat{q}_{j}+F\left(\hat{q}_{j}\right)\right]
$$

again for $i=1, \ldots n$. Now we sum both sides over $i$, which yields 
where $Q$ is defined as the average frequency of $\mathrm{C}$ individuals in the whole population at equilibrium, i.e.,

$$
Q=(1 / n) \sum_{i=1}^{n} \hat{q}_{i}
$$

To determine whether conformist transmission is adaptive we need to calculate the equilibrium frequency of $\mathrm{C}$ in the various subpopulations, $\hat{q}_{1}, \ldots, \hat{q}_{n}$. The right side of (8) defines a function of $\hat{q}_{i}$ which we will label $f\left(q_{i}\right)$ and which is plotted in Fig. 2 for two different combinations of values of $U_{i}, A$, and $m$. Equation (8) says that the $i$ th subpopulation will be in equilibrium if $Q=f\left(\hat{q}_{i}\right)$. In general, $Q$ will also be a function of $\hat{q}_{i}$. If, however, we assume that there are a large number of subpopulations, then the average frequency of $\mathrm{C}$ individuals in the whole population will be approximately independent of the changes in the frequency in any subpopulation. Thus for any given value of $Q$, say $\mathrm{Q}^{*}$, the equilibrium frequency of $\mathrm{C}$ in the $i$ th subpopulation, $\bar{q}_{i}$, can be determined graphically by finding intersection $F\left(\hat{q}_{i}\right)$ and the line $Q=Q^{*}$. As is shown in Fig. 3, there may be more than one possible equilibrium.

So far the presentation has been fairly general. Now we make the special assumption that $Q=0.5$, which will occur if the distribution of $U_{i}$ is symmetric around zero. (This will occur if the distributions of $\mathrm{V}_{\mathrm{i}, \mathrm{C}}$ and $\mathrm{V}_{\mathrm{i}, \mathrm{D}}$ are identical and if the distribution of initial frequencies of $\mathrm{C}$ in the subpopulations satisfies certain conditions.) With this special assumption it is easy to show that conformist transmission is adaptive. In Fig. 2 the form of $f\left(\hat{q}_{i}\right)$ is plotted, assuming that $A=0$ and that $U_{i}<0$. The latter assumption means that $\mathrm{D}$ individuals are favored by selection. Notice that $\hat{q}_{i}<0.5$, i.e., at equilibrium the favored trait is more frequent in every subpopulation. This means that offspring who acquire their behavior via a

s(continued)

$$
\begin{aligned}
0 & =-m \sum_{i=1}^{n \cdot} \hat{q}_{i}+(1-m) \sum_{i=1}^{n} F\left(\hat{q}_{i}\right)+(m / n) \sum_{i=1}^{n} \sum_{j=1}^{n}\left[\left(\hat{q}_{j}+F\left(\hat{q}_{j}\right)\right]\right. \\
& =-m \sum_{i=1}^{n} \hat{q}_{i}+(1-m) \sum_{i=1}^{n} F\left(\hat{q}_{i}\right)+m \sum_{j=1}^{n} F\left(q_{j}\right)+M \sum_{i=1}^{n} F\left(q_{j}\right) \\
& =\sum_{j=1}^{n} F\left(q_{j}\right)
\end{aligned}
$$

Thus at equilibrium the changes in the frequency of $\mathrm{C}$ before dispersal must sum to zero. With this result Eq. $\left(^{*}\right)$ above becomes Eq. (8) in the text. 


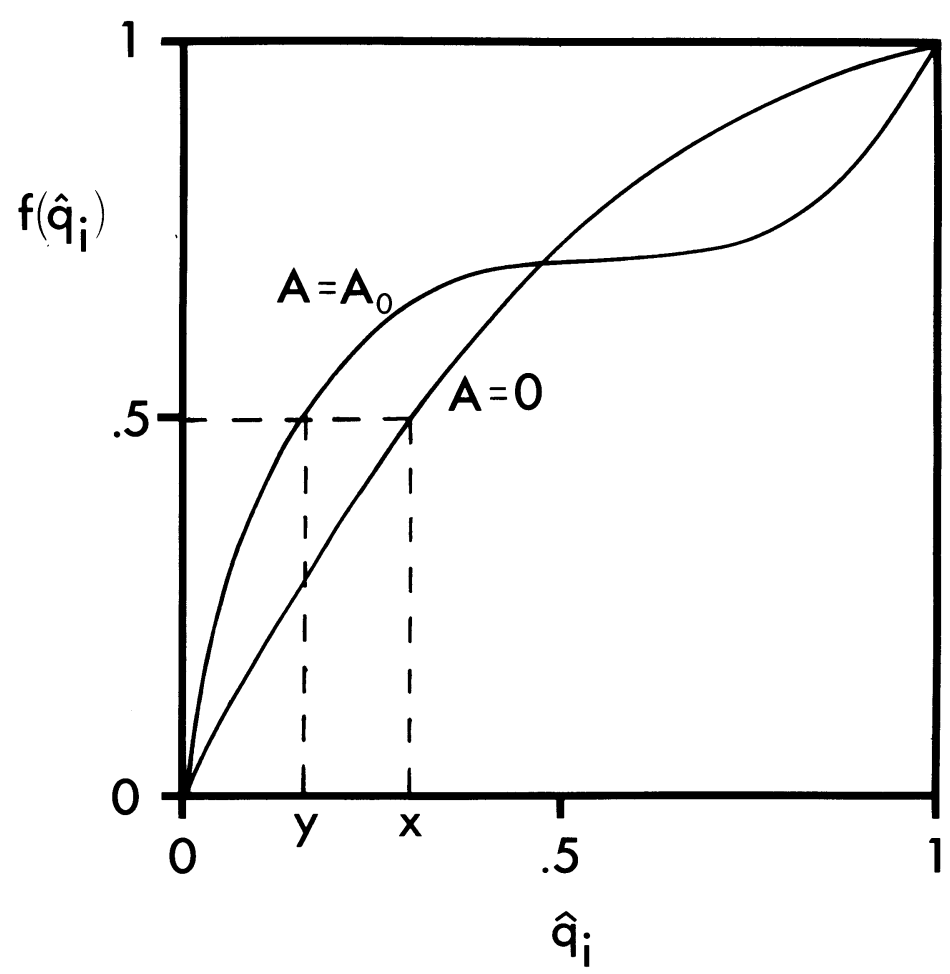

Fig. 2. This figure plots $f\left(\hat{q}_{i}\right)$ for $A=0$ and $A=A_{0}>0$ (the actual parameter values used were $U_{i}=-0.05, m=0.05$, and $A_{0}=0.1$ ), and shows equilibrium frequencies in the $i$ th subpopulation under the assumption that $Q$ $=0.5$ for $A=0$ and $A=A_{0}$. These values are labeled $x$ and $y$, respectively.

conformist cultural inheritance rule have a higher probability of acquiring the trait favored by selection than offspring who utilize a linear rule. Thus selection should act to increase $A$. In Fig. $2, f\left(\hat{q}_{i}\right)$ is also plotted assuming that $A$ takes on a positive value, say $A_{0}$. Notice that $\hat{q}_{i}$ is even smaller than in the previous case and therefore selection will favor individuals with even a stronger conformist effect (i.e., whose cultural transmission rule is characterized by $A>A_{0}$ ).

Selection will continue to increase $A$ until $A=1$ or a situation like that pictured in Fig. 3 is reached. Now there are three equilibrium values of $\hat{q}_{i}$, which we will label $\hat{q}_{C}, \hat{q}_{T}$, and $\hat{q}_{D}$. The central equilibrium $\hat{q}_{T}$ is unstable, but the other two are stable. In subpopulations at equilibrium at $\hat{q}_{C}$, selection will continue to favor increased values of $A$, while in subpopulations at equilibrium at $\hat{q}_{D}$, selection will favor decreased values of A. Determining which of these two processes will predominate is beyond the scope of this paper. It is possible 


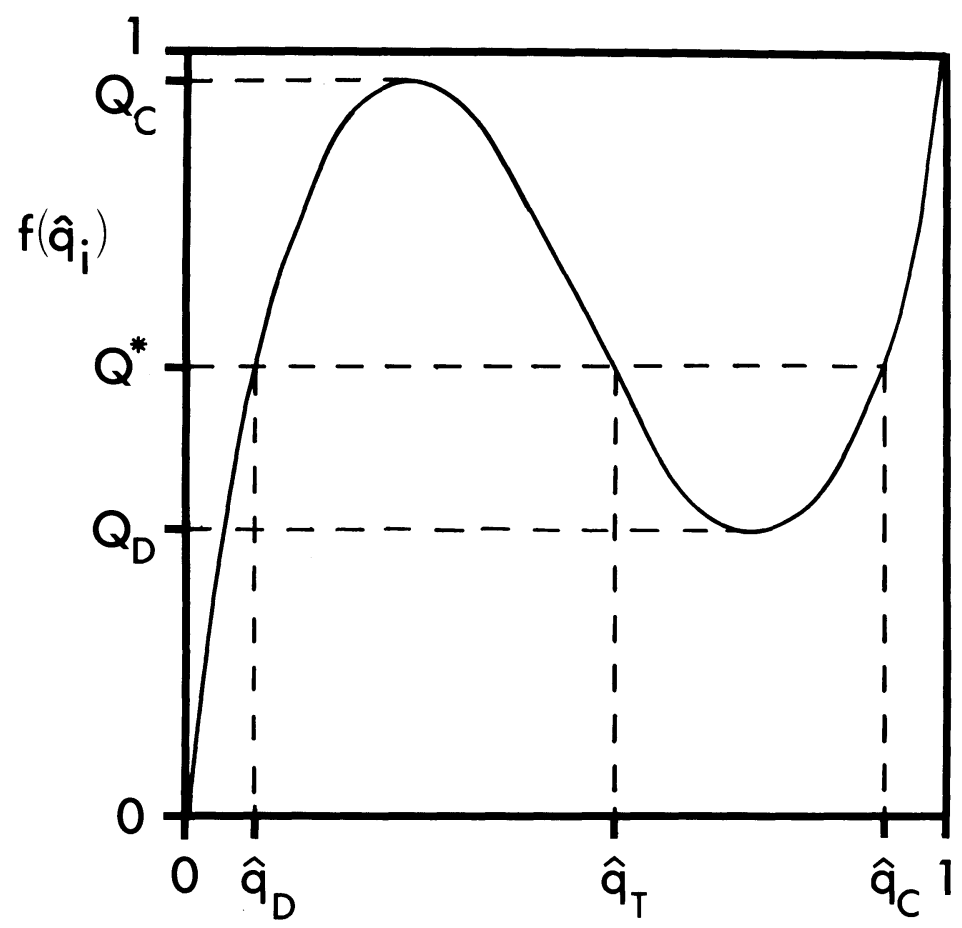

Fig. 3. This figure shows $f\left(\hat{q}_{i}\right)$ as a function of $q$ for the parameter values $U$ $=-0.05, m=0.05$, and $A=0.35$. For $Q=Q^{*}$, the three equilibria possible in subpopulation $i-\hat{q}_{C}, \hat{q}_{T}$, and $\hat{q}_{D}$ - are as shown.

to show, however, that selection may continue to favor a stronger conformist effect even in the situation pictured in Fig. 3.

This special example illustrates what we believe is an important property of conformist transmission. In spatially varying environments, it can serve as a simple general rule that increases the probability that individuals acquire traits that are favored in the local habitat. Thus it is reasonable to suppose that selection might favor cultural transmission rules with a strong conformist effect, much as it is reasonable to assume that selection will favor direct biases that, on average, increase genetic fitness. In the next section we will examine what happens when traits acquired via conformist cultural transmission have effects on the persistence of the subpopulation.

\section{CULTURAL GROUP SELECTION}

The sociobiologist's prediction that human behavior should be egoistic is based on the belief that group selection is not an important force in evolution. 
The basic model of group selection is as follows. ${ }^{6}$ As in the previous section, one assumes that the population is subdivided into a large number of subpopulations or demes, each partially isolated from the rest, receiving only a relatively small number of immigrants in each generation. In the simplest models it is assumed that there are two traits, cooperative (C) and noncooperative ( $\mathrm{D}$, for "defector"), and that the cooperative trait is at a selective disadvantage in each deme, so that selection within the deme acts to decrease the frequency of the cooperative trait. At the same time, increasing the frequency of the noncooperative trait is assumed to increase the probability the the deme will become extinct and be recolonized by individuals drawn from the rest of the population, and therefore selection between groups acts to increase the frequency of cooperators in the population. The strength of the group selection process will depend on the rate of extinction as a function of the frequency of cooperators and the variation between demes in the frequency of cooperators.

The majority of evolutionary biologists currently believe that "individual" selection, i.e., selection within demes, is a much stronger force than group selection. This belief is largely based on theoretical studies that indicate that group selection can be effective only if demes are very small, extinction rates are very high, and migration rates are very low (e.g., Eshel, 1972; Levin and Kilmer, 1974; Maynard Smith, 1976; Slatkin and Wade, 1978; see Wade, 1978, and Uyenoyama and Feldman, 1980, for reviews). It is felt that this combination of conditions is unlikely to be found in nature. Intuitively, the main reason that group selection is a weak force is that selection and dispersal are constantly acting to destroy the variability between groups, which is necessary for group selection to be effective. Sampling error caused by finite populations and by the recolonization process creates between-group variability. The models show that these sources of variability are insufficient unless the conditions listed above hold.

The theoretical argument against group selection is based on models that assume genetic inheritance. The same conclusions may not hold for culturally

${ }^{6}$ In recent years there has been a revival of interest in group selection as an explanation for the evolution of altruistic behaviors. Population structures that lead to assortive interaction of altruistic individuals are necessary for altruistic behaviors to be favored. There is considerable controversy, however, about the logical relationship between different population structures and their importance in nature. Some authors (e.g., Maynard Smith, 1976; Dawkins, 1980) argue that "kin selection" is categorically distinct from group selection, and that only kin selection is likely to be important in nature, while others (e.g., Hamilton, 1975; Wade, 1978; Uyenoyama, 1979; Wilson, 1980) argue that kin and group selection represent two ends of a continuum of processes, and that a variety of different processes may be of considerable importance. There is a tendency for the latter author to use the term group selection to refer to all processes that involve differences in the mean fitness of groups. Here, we use the term group selection to refer to the process described in the text. By doing this, we do not mean to imply that the "intrademic" group selection processes studied by Wilson, Wade, and others are unimportant. 
transmitted traits. In this section we analyze a model of group selection acting on a cultural trait which suggest that conformist transmission can cause variation between demes to be substantial compared to variation within demes. In some circumstancés, group selection can be a strong force in determining the eventual equilibrium of the population, even when demes are arbitrarily large, extinction rates are small, and migration rates are substantial.

We make two modifications to the model presented in the previous section. First, we assume that $U_{i}=U<0$ in every deme. This means that noncooperators ( $\mathrm{D}$ individuals) are favored by natural selection and direct bias in every deme. Second, we assume that occasionally one of the subpopulations becomes extinct, leaving an empty habitat which is then recolonized by individuals from the surviving subpopulations. The probability that the $i$ th subpopulation becomes extinct during generation $t$ is assumed to be a monotonically decreasing function of $q_{i, t}$, labeled $E\left(q_{i, t}\right)$. Put another way, increasing the proportion of defectors in a subpopulation increases the probability that the subpopulation becomes extinct. For the present, this extinction may be thought of as involving the actual deaths of the individuals in the extinct population. We will argue in the discussion, however, that "cultural extinction" need not involve physical deaths.

Under what conditions will group selection be stronger than individual selection? To answer this question we first determine the equilibrium of the population in the absence of any extinctions, and then assume that extinctions occur sufficiently infrequently for the populations as a whole to reach equilibrium before the next extinction occurs. This combination of very large subpopulations and very infrequent extinction weakens the action of group selection, and in genetic models would result in group selection being of negligible importance.

Next we determine if (1) an equilibrium composed exclusively of defectors is unstable, and (2) an equilibrium composed exclusively of cooperators is stable. If both these conditions are satisfied, we will say that group selection is a strong force. Suppose that the values of $A, U$, and $m$ are such that the equilibrium of the $i$ th population can be determined using Fig. 3. Further, imagine that all of the subpopulations except subpopulation $i$ are at equilibrium, and that the frequency in the whole population is $Q$. In this case, the equilibrium of the $i$ th subpopulation depends on its initial frequency. If the initial frequency is greater than $\hat{q}_{T}$, the subpopulation will reach a stable equilibrium with cooperators at a high frequency $\hat{q}_{C}$. We will refer to. such subpopulations as "cooperator equilibria." If the initial frequency of cooperators is less than $\hat{q}_{T}$, the subpopulation will achieve a stable "defector equilibrium" with a low frequency of cooperators $\hat{q}_{D}$.

These results about the equilibrium of a single subpopulation can be used heuristically to determine the equilibrium for the entire population. First, note that conditions in each of the subpopulations are identical. Thus 
all of the subpopulations must be in either one or two equilibrium states. The function $f\left(\hat{q}_{i}\right)$ has an internal minimum labeled $Q_{D}$ and an internal maximum labeled $Q_{C}$. If the mean frequency of cooperators $Q$ in the whole population is greater than $Q_{C}$ or less than $Q_{D}$, then only one stable subpopulation exists at equilibrium, and therefore all subpopulations must be at the same frequency. Label this frequency $\hat{q}^{*}$. Since all subpopulations are identical, the entire population can be in equilibrium only if $f\left(\hat{q}^{*}\right)=\hat{q}^{*}$. This occurs only for $\hat{q}^{*}=1$ and $\hat{q}^{*}=0$. Thus, for $Q<Q_{D}$, the only possible stable equilibrium is the entire population composed entirely of defectors. If $Q_{C}>Q>Q_{D}$, then two stable equilibria are possible, $\hat{q} C(Q)$ and $\hat{q}_{D}(Q)$. Let $p$ be the proportion of the subpopulations that are at the cooperative equilibrium, $\hat{q}_{C}(Q)$. An internal equilibrium for the entire population will exist if

$$
p \hat{q}_{C}(Q)+(1-p) \hat{q}_{D}(Q)=\mathrm{Q}
$$

Satisfying condition (10) does not assure that an equilibrium is stable or that it can be reached from any particular initial condition. Computer simulations indicate, however, that such equilibria are stable once they are reached, and that most initial configurations for which $Q_{C}>(1 / n) \sum_{i=1}^{n} q_{i . t}$ $>Q_{D}$ result in an internal equilibrium of the kind described by Eq. (10). These simulations also indicate that (8) gives very good approximations for the values of $\hat{q}_{C}$ and $\hat{q}_{D}$, even when the number of subpopulations is as small as 10 .

The parameters $m, A$, and $U$ affect the shape of $f\left(\hat{q}_{i}\right)$ in the following way: increasing $A$ and decreasing $m$ both have the effect of increasing $Q_{C}$ and decreasing $Q_{D}$. This increases the range of values of $Q$ for which there is an internal equilibrium. For sufficiently large $A$ or small $m, Q_{D}<0$. Since $Q_{C}>1-Q_{D}$, this ensures that internal equilibria are possible for all values of $Q$. Increasing $A$ also has the effect of moving $\hat{q}_{T}$ closer to onehalf, thereby increasing the range of initial frequencies of cooperators that result in an equilibrium at $\hat{q}_{C}$. Finally, increasing $U$ increases $Q_{C}$ and $Q_{D}$ and moves $\hat{q}_{T}$ closer to 1 .

Now consider the effect of an occasional rare extinction on a population that has achieved an internal equilibrium. A fraction $p$ of the subpopulations are assumed to be an internal equilibrium $\hat{q}_{C}$ and a fraction $(1-p)$ are at $\hat{q}_{D}$. The internal unstable equilibrium is $\hat{q}_{T}$. The extinction of a subpopulation leaves an empty habitat that is colonized by individuals from the other subpopulations. After extinction and colonization, both the subpopulation and the population as a whole will move toward a stable 
equilibrium. This equilibrium may not be identical to the one that existed before the extinction. Suppose a cooperator subpopulation becomes extinct and is replaced at the new equilibrium by a defector subpopulation. This changes the mean frequency of cooperators from $Q$ to a slightly smaller value. This in turn changes the possible stable equilibria to slightly lower values and reduces the frequency of cooperator subpopulations after extinction. If a defector subpopulation becomes extinct and is replaced by a cooperative one, then a new equilibrium is reached, characterized by slightly increased values of these quantities.

What is the long-run net effect of changes caused by extinctions on the evolution of the entire population? The eventual equilibrium in the subpopulation undergoing colonization is determined by the frequency of cooperators among the colonizers, $q_{k}$. Let $h\left(q_{k}\right)$ be the probability density function for $q_{k}$. Then the probability that $q_{k}$ is greater than $x$ is given by the function $H(x)$, where $H(x)=\int_{\mathrm{x}}^{1} h\left(q_{k}\right) d q_{\mathrm{k}}$. If $q_{\mathrm{k}}<\hat{q}_{T}$, then the population will move toward a defector equilibrium. If $q_{k}>\hat{q}_{T}$, the new equilibrium will be cooperative. Let $p^{\prime}$ be the fraction of cooperative subpopulations after the extinction. The expected value of $p^{\prime}, E\left(p^{\prime}\right)$, is given by

$$
E\left(p^{\prime}\right)=p\left[1-E\left(q_{C}\right)\right]+p E\left(q_{C}\right) H\left(q_{T}\right)+(1-p) E\left(q_{D}\right) H\left(q_{T}\right)
$$

The fraction of cooperative subpopulations may be expected to increase if

$$
H\left(\hat{q}_{T}\right)>\frac{p E\left(\hat{q}_{C}\right)}{p E\left(\hat{q}_{C}\right)+(1-p) E\left(\hat{q}_{D}\right)}
$$

The right side of (12) gives the fraction of all extinctions that occur in subpopulations in equilibrium at a value $\hat{q}_{C}$. Since we assume that the extinction rate is a monotonically decreasing function of the frequency of cooperators, this quantity is always less than $p$. The left side of (12) is the probability that a habitat recently vacated by extinction will achieve a cooperative equilibrium after recolonization. Thus, if the probability that a cooperative subpopulation will be formed after an extinction exceeds the probability that cooperative subpopulations become extinct (for a given value of $p$ ), then the frequency of cooperators in the whole population will increase.

The evolution of the population as a whole depends critically on the magnitude of $H\left(\hat{q}_{T}\right)$, which in turn depends on the mode of colonization. To see this, first assume that each colonizing individual is drawn at random from the whole population, and that the number of colonizers is large. In this case, a fraction $Q$ of colonizers are cooperators. Therefore, 
$H(x)$ has the form

$$
H(x)=\left\{\begin{array}{l}
1 \text { if } x>Q \\
0 \text { if } x<Q
\end{array}\right.
$$

Thus, if $Q>\hat{q}_{T}, p$ increases. Recall, however, that $\hat{q}_{T}$ is a function of $Q$. It can be shown that $Q$ is always less than $\hat{q}_{t}$, and thus that cooperation can never increase. Now, assume that a large group of colonizers is drawn from a single randomly chosen subpopulation. In this case, there is a probability $p$ that the frequency of cooperators among the colonizers will be $\hat{q}_{C}$, and a probability $1-p$ that the frequency of cooperators among the colonizers will be $\hat{q}_{D}$. Since we know $\hat{q}_{C}>\hat{q}_{T}>\hat{q}_{D}$, this means that $H\left(\hat{q}_{T}\right)=p$. In this case (12) becomes

$$
E\left(\hat{q}_{C}\right)>p E\left(\hat{q}_{C}\right)+(1-p) E\left(\hat{q}_{D}\right)
$$

which is satisfied for all internal equilibria. When colonizers are drawn from a single subpopulation, the frequency of cooperative subpopulations will increase whenever $E\left(\hat{q}_{C}<E\left(\hat{q}_{D}\right)\right.$. Remarkably, this condition is independent of the magnitude of the difference between $E\left(\hat{q}_{C}\right)$ and $E\left(\hat{q}_{D}\right)$. As long as conformist transmission is strong enough (relative to individual selection and biased transmission) to create two subpopulation equilibria, the relative strengths of group selection and individual selection are irrelevant; only the extinction rate as a function of $\hat{q}_{i}$ is important. As long as $E(\cdot)$ is decreasing function of $\hat{q}_{i}$, cooperation will go to fixation once it is established.

Clearly, much more complex models of colonization are possible. The number of subpopulations contributing colonizers could vary according to some probability distribution. The absolute number of colonizers might also be small and variable, and colonizers might not represent an unbiased sample of the population. We cannot deal with these complexities here. Instead, we will assume the following model. Let $g(j)$ give the probability that colonizers are drawn at random from $j$ subpopulations $(j=1, \ldots, n)$. Then for small values of $p$,

$$
H\left(\hat{q}_{T}\right) \approx g(1) p
$$

In this case, (12) reduces to the following expression:

$$
g(1)>E\left(\hat{q}_{C}\right) / E\left(\hat{q}_{D}\right)
$$


Inequality (16) says that cooperators can increase in the population when rare if the ratio of the extinction rate of cooperative subpopulations to that of defector subpopulations is less than the probability that colonizers are drawn from a single subpopulation. By symmetry the condition for $p$ to increase when $(1-p)$ is small is

$$
g(1)<E\left(\hat{q}_{D}\right) / E\left(\hat{q}_{C}\right)
$$

This condition is satisfied whenever (16) is satisfied. Whenever extinction and recolonization make $p=0$ un unstable equilibrium, $p=1$ is stable equilibrium. It is important to remember that (16) and (17) require that $A$, $U$, and $m$ are such that both cooperative and noncooperative equilibria exist for all values of $Q$. Given that this is the case, however, these conditions are nearly independent of the relative magnitudes of $U$ and $E(\cdot)$. Conformist transmission "decouples" the processes of individual and group selection near the boundaries $p=0$ and $p=1$. This will not be true for other values of $p$. Here $H$ will depend on $\hat{q}_{T}$, which in turn depends on the relative magnitudes of $U, A$, and $m$.

Another remarkable feature about (16) and (17) is that they depend on the ratio of the extinction rates. In conventional models, the strengths of both group and individual selection depend on the ratios of survival rates of groups and individuals. This difference is important because the probabilities of extinction might be very low, and at the same time the ratio $E\left(\hat{q}_{C}\right) / E\left(\hat{q}_{D}\right)$ might be much less than 1 . For example, suppose that the survival rate of cooperative subpopulations is 0.9999 and that of noncooperative subpopulations is 0.99 . The ratio of the extinction rates is 0.01 , and therefore cooperation will increase if only $1 \%$ of the empty habitats is colonized by a single subpopulation.

We have been unable to derive general conditions under which the combination of conformist transmission and group selection would favor the increase of cooperators. The heuristic argument outlined above suggests, however, that the following conditions favor the establishment of cooperators in the population:

1. The parameters $A, U$, and $m$ must be such that both cooperator and defector equilibria can coexist when cooperators are rare. This requires that the conformist effect be substantially stronger than selection and migration. The formal condition is $(1-A / U)^{2} / 8(A / U) \cdot>(m / U)$.

2 . The mode of colonization should lead to a significant probability that colonizers are drawn from a single subpopulation. 
3. The ratio of the extinction rates of cooperator and defector subpopulations should be significantly less than 1 , but the extinction rates need not be large.

Given that conformist transmission is a significant force acting on the frequency of traits within subpopulations, and that the mode of colonization is favorable, group selection can increase the frequency of cooperators. This can occur even if extinction rates are very low, migration rates are substantial, and subpopulations are very large. Without conformist transmission, selection and migration constantly erode the variation between subpopulations. Extinction must be frequent enough to balance this process. Strong conformist transmission creates the possibility that subpopulations may be maintained indefinitely with a higher frequency of cooperators. Whether cooperators then increase depends on the relative extinction rates of cooperator and defector subpopulations, and the probability that recolonized subpopulations achieve a cooperative equilibrium.

\section{DISCUSSION}

The empirical facts of human cooperation are not easily reconciled with the view that human nature is fundamentally egoistic. Alternative views that hold that humans are by nature cooperative face two related difficulties. First, how can we account for the evolution of humans who cooperate on a large scale with unrelated individuals? Second, given that individuals belong to many different groups with potentially conflicting goals, with which group (or groups) will an individual identify? The model of cultural group selection provides one possible answer to these questions.

The main requirement of the model is that humans inherit at least some portion of the values, goals, and beliefs that determine their choices by way of conformist cultural transmission. To explain the evolution of human cooperation, we need to account for the evolution of a human capacity for culture which is characterized by the conformist effect. We have seen that conformist transmission may be favored in spatially variable environments because it provides a simple, general rule that increases the probability of acquiring behaviors favored in the local habitat. It is plausible that this effect could compensate for what is, from the genes" "point of view," the excessive cooperation that also may result from conformist transmission.

To deal with the question of conflicting goals, we must interpret the results of our very simple model in terms of the complexity of social reality. Under specified conditions group selection favored cooperators, individuals whose behavior reduced their own welfare but increased the probability that 
their subpopulation would survive each generation. The key defining characteristic of the subpopulation is that it is completely mixed; sets of cultural parents are drawn at random from the pool of individuals within it. In contrast, the flow of cultural traits between subpopulations is restricted, since migration rates are less than 1 . We shall say that the subpopulation is "culturally endogamous." In human societies, individuals can belong to many social groupings, of varying sizes and purposes, and with varying criteria for membership. Our model suggests that humans will engage in behaviors that promote the interests of a particular groups as a whole only if (1) they acquire behaviors culturally from other members of the group via conformist cultural transmission, and (2) the group is culturally endogamous. These characteristics, taken together, specify the group with which individuals should identify.

Different social groupings may be culturally endogamous for some traits but not for others. For traits that are acquired by young children from members of their family, the culturally endogamous group might be very similar to the genetic deme. That is, an individual's cultural parents would be drawn from the same social grouping as its genetic parents. The culturally endogamous group for a trait acquired disproportionately from parents of one sex, may be different from the culturally endogamous group for traits acquired from parents of both sexes. For example, suppose that behavior during warfare is acquired exclusively from males. Such behavior could include rules for the formation of alliances, as well as actual combat techniques. In patrilocal societies, the culturally endogamous group for behavior during warfare could be very small (so small as to require substantial amendments to the model). In the same societies, the culturally endogamous group for behaviors acquired from both sexes - for example, language or religious belief - could be very large. In contrast, in matrilocal societies, the culturally endogamous group might be the same for warfare, language, and religion. For traits acquired as an adult, the culturally endogamous group may be different again. For example, many aspects of individual behavior in modern corporations, including professional goals, work norms, and beliefs about the nature of the product and the marketplace, are acquired culturally from individuals who precede them in the firm (Van Maanen and Schein, 1979). For these behaviors, the culturally endogamous group is the firm. Other examples of groups that may be culturally endogamous for certain traits include fraternal organizations, craft guilds, and, of course, academic disciplines.

When there is conformist transmission, the extinction of a group need not entail the physical death of individuals; the breakup of the group as a coherent social unit and the dispersal of its members to other groups will suffice. Imagine that the members of an "extinct" subpopulation are 
dispersed randomly to all the other subpopulations. Because the members of a subpopulation are either mostly cooperators or mostly defectors, this will change the frequency of cooperators in the pool of migrants during the generation in which the extinction takes place. This will perturb each of the subpopulations from its equilibrium value, but since each subpopulation is small compared to the whole, the perturbation will be small. Each subpopulation will reach the same equilibrium that it would have reached if the members of the extinct group had been removed from it. Intuitively, the dispersal of a group is equivalent to extinction because conformist transmission favors the more common variant. Cooperators persist in cooperative groups because they are common in those groups. If they are dispersed, their numbers will usually be insufficient to cause the frequency of cooperators in defector groups to exceed the threshold necessary to cause a change to a cooperative equilibrium.

To put this in more concrete terms, consider the following hypothetical example. Suppose that the cultural trait in question affects the rotation period in a swidden agricultural system. Defectors have a cultural variant that causes them to have a shorter rotation period. Occasionally, when coupled with especially unfavorable weather, this causes a temporary failure of the agricultural system in the subpopulation. It is unlikely that this would lead to the actual extinction of the group. Rather, members disperse, different families attempting to joint other groups. In these new groups, the immigrants form only a small proportion of the group, so that, if conformist transmission is important, they will have little effect on group behaviors. Clearly, this is only one of many patterns of dispersal that might actually take place. Different patterns will lead to different outcomes. For another example, involving a reinterpretation of Rappaport's (1968) study of the Maring, see Peoples (1982).

The model suggests that group selection is more effective when vacant habitats are recolonized by individuals drawn from a single subpopulation. This model of colonization seems plausible in the human case for several reasons: first, in a social species in which division of labor and cooperative subsistence activities are important, it seems likely that individuals will immigrate as a social unit to colonize empty habitats. Second, even if a vacant habitat is colonized by groups that originated in more than one subpopulation, behavioral isolating mechanisms may prevent them from fusing to form a new culturally endogamous group. Finally, warfare may play an important role in determining group survival. Defeated groups may be dispersed and replaced by individuals from the victorious group.

One human grouping that seems generally to satisfy the requirements of the model is the ethnic group. The flow of cultural traits within the ethnic group is often much greater than the flow between ethnic groups. The model predicts that group selection acting on culturally transmitted traits 
Table II. List of Behaviors Defining the Syndrome of Ethnocentrism ${ }^{a}$

\begin{tabular}{|c|c|c|c|}
\hline \multicolumn{2}{|c|}{$\begin{array}{l}\text { Attitudes and behaviors } \\
\text { toward ingroup }\end{array}$} & \multicolumn{2}{|r|}{$\begin{array}{l}\text { Attitudes and behaviors } \\
\text { toward outgroup }\end{array}$} \\
\hline 1.1 & $\begin{array}{l}\text { See selves as virtuous } \\
\text { and superior }\end{array}$ & 1.2 & $\begin{array}{l}\text { See outgroup as } \\
\text { contemptible, immoral, } \\
\text { and inferior }\end{array}$ \\
\hline 1.3 & $\begin{array}{l}\text { See own standards of } \\
\text { value as universal, in- } \\
\text { trinsically true. See } \\
\text { own customs as orig- } \\
\text { inal, centrally human }\end{array}$ & & \\
\hline 1.4 & See selves as strong & $\begin{array}{l}1.5 \\
1.6 \\
1.7\end{array}$ & $\begin{array}{l}\text { See outgroup as weak } \\
\text { Social distance } \\
\text { Outgroup hate }\end{array}$ \\
\hline 1.8 & $\begin{array}{l}\text { Sanctions against } \\
\text { ingroup theft }\end{array}$ & 1.9 & $\begin{array}{l}\text { Sanctions for outgroup } \\
\text { theft or absence of } \\
\text { sanctions against }\end{array}$ \\
\hline 1.10 & $\begin{array}{l}\text { Sanctions against } \\
\text { ingroup murder }\end{array}$ & 1.11 & $\begin{array}{l}\text { Sanctions for outgroup } \\
\text { murder or absence of } \\
\text { sanctions against } \\
\text { outgroup murder }\end{array}$ \\
\hline 1.12 & $\begin{array}{l}\text { Cooperative relations } \\
\text { with ingroup members }\end{array}$ & 1.13 & $\begin{array}{l}\text { Absence of cooperation } \\
\text { with outgroup members }\end{array}$ \\
\hline 1.14 & $\begin{array}{l}\text { Obedience to ingroup } \\
\text { authorities }\end{array}$ & 1.15 & $\begin{array}{l}\text { Absence of obedience to } \\
\text { outgroup authorities }\end{array}$ \\
\hline 1.14 & $\begin{array}{l}\text { Willingness to remain } \\
\text { an ingroup member }\end{array}$ & 1.17 & $\begin{array}{l}\text { Absence of conversion } \\
\text { to outgroup membership }\end{array}$ \\
\hline \multirow[t]{5}{*}{1.18} & $\begin{array}{l}\text { Willingness to fight } \\
\text { and die for ingroup }\end{array}$ & 1.19 & $\begin{array}{l}\text { Absence of willingness } \\
\text { to fight and die for } \\
\text { outgroups }\end{array}$ \\
\hline & & 1.20 & $\begin{array}{l}\text { Virtue in killing } \\
\text { outgroup members in } \\
\text { warfare }\end{array}$ \\
\hline & & 1.21 & $\begin{array}{l}\text { Use of outgroups as bad } \\
\text { examples in the training } \\
\text { of children }\end{array}$ \\
\hline & & 1.22 & $\begin{array}{l}\text { Blaming of outgroup for } \\
\text { ingroup troubles }\end{array}$ \\
\hline & & 1.23 & $\begin{array}{l}\text { Distrust and fear of the } \\
\text { outgroup }\end{array}$ \\
\hline
\end{tabular}

${ }^{a}$ From LeVine and Campbell (1972).

will favor cooperative behavior within ethnic groups and noncooperative behavior toward members of other groups. Table II lists the traits identified with the syndrome of ethnocentrism in LeVine and Campbell (1972). In their book, LeVine and Campbell review the evidence that this syndrome is a very common characteristic of human ethnic groups and the theories that have been advanced to explain it. The list of behaviors in Table II seems consistent with the predictions of the model. Sanctions against theft and murder within the group provide civil order, a public good benefiting group members. This contrasts with the lack of sanctions protecting outgroup 
members. Moreover, cooperative behavior typifies interactions between group members, and lack of cooperation typifies interactions between members of different groups. Finally, individuals are willing to fight and die for their own group in warfare against other groups. In recent times, actions on behalf of the ethnic group have often been taken in direct opposition to the authority and power of the modern state-witness movements for ethnic autonomy in many parts of the world today. It also must be kept in mind that the groups in question are often very large. In such large groups, it is hard to imagine that any kind of reciprocal arrangement is responsible for the observed interaction. These generalization about human ethnocentrism suggest that the ethnic group is one locus of altruistic behavior.

The variation in behavior toward ethnic group members also provides some support for the hypothesis that group selection acting on culturally transmitted behavior has shaped human behavior. LeVine and Campbell categorize social structures as "socially divisive" and "socially integrative": "The former type of society has structural features such as patrilocality or local group endogamy that foster the development of a parochial loyalty structure. . . . In socially integrative types, dispersion of males through the means discussed above fosters the development of loyalities to wider groupings.... Since socially divisive societies have warfare among segments of the ethnic community and socially integrated societies do not, the social structures are seen as favoring different norms of conduct concerning social intercourse within the ethnic community" (p. 53, emphasis ours). They go on to argue that while socially divisive societies are characterized by extensive feuding and violence, they are infrequently involved in large-scale warfare, and when they are involved in warfare, alliances are formed opportunistically on the basis of immediate military contingencies. In contrast, while socially integrative societies have much less violence within groups, they readily cooperate in large-scale conflict. LeVine and Campbell cite Noberini's (1966) cross-cultural study of warfare as supporting this view. Again, it appears that the unit upon which group selection has worked is the culturally endogamous group. If this unit is small, as in the case of socially divisive societies, then so is the unit within which social cooperation takes place. In socially integrated societies, the culturally endogamous unit is larger (at least with regard to traits transmitted by men) and so is the scale of violent conflict.

It is useful to contrast the predictions of the cultural group selection hypothesis with those that result from egoistic views of human nature. Economic Man and the Sociobiological Man ought to be blind to ethnicity when making decisions about military alliances. Economic Man's choice ought to be based on calculation of expected chance of victory. Sociobiological Man might include in his or her calculations con- 
sideration of genetic relatedness. Both might opt to support individuals from their local group with whom they had reciprocal ties. It is hard to see why they should regularly ally with large groups of co-ethnics with whom they have no genetic or reciprocal interest.

The simple model of cultural group selection outlined here is not, strictly speaking, verified by the data concerning ethnic cooperation. Nor is it a complete hypothesis to account for cooperative behavior in humans. The real world is undoubtedly much more complex than we have allowed. However, the model does illustrate what we believe is a crucial property of the evolution of cultural species: if the rules of cultural transmission are different from the rules of genetic transmission, similar selective regimes may result in very different equilibria. The model also provides qualitative predictions about the kind of transmission rules that might explain human cooperative behavior, i.e., a cultural transmission rule that increases the frequency of the more common variant can cause group selection to be a strong force in determining the kinds of behaviors that characterize different human societies.

A variety of important theoretical questions are yet to be resolved; more important, however, are the empirical questions. Theoretical exercises such as this can help to improve our understanding of the relationship between the mode of cultural transmission and the properties of the resulting evolutionary process. Soon, however, we will require data about the details of cultural transmission. We need to know, for example, under what circumstances (if any) human cultural transmission is conformist, and from whom individuals acquire particular behavioral traits. We believe that such questions are answerable, and that social psychologists, sociologists, and anthropologists will provide the answers.

\section{ACKNOWLEDGMENTS}

We would like to thank Donald T. Campbell for extensive and insightful discussions of the ideas contained in this paper, and James Peoples for useful comments on an earlier draft.

\section{REFERENCES}

Alexander, R. D. (1979). Darwinism and Human Affairs. Univ. Washington Press, Seattle. Axelrod, R. (1980a). Effective choice in the prisoner's dilemma. Journal of Conflict Resolution 24: 3-25.

Axelrod, R. (1980b). More effective choice in the prisoner's dilemma. Journal of Conflict Resolution 24: 379-404. 
Axelrod, R., and Hamilton, W. D. (1981). The evolution of cooperation. Science 211: 13901396.

Boyd, R., and Richerson, P. J. (1980). Sociobiology, culture, and economic theory. Journal of Economic Behavior and Organization 1: 97-121.

Boyd, R., and Richerson, P. J. (1981) Culture, biology, and the evolution of variation between human groups. In Collins, M. S., Wainer, I. W., and Bremner, T. A. (eds.), Science and the Question of Human Equality, Westview Press, Boulder, Colo., pp. 99-152.

Buchanan, J. (1968). The Supply and Demand of Public Goods. Rand-McNally, Chicago.

Campbell, D. T. (1965). Variation and selective retention in sociocultural evolution. In Barringer, H. R., et al. (eds.), Social Change in Developing Areas. Shenkman, Cambridge, pp. 19-49.

Campbell, D. T. (1975). On the conflicts between biological and social evolution and between psychology and moral tradition. American Psychologist 30: 1103-1126.

Cavalli-Sforza, L. L., and Feldman, M. W. (1981). Cultural Transmission and Evolution. Princeton Univ. Press, Princeton, N.J.

Dawkins, R. (1980). Twelve misunderstandings of kin selection. Zeitschrift für Tierpsychologie 51: 184-201.

Durham, W. H. (1976). The adaptive significance of cultural behavior. Human Ecology 4: 89-121.

Eshel, I. (1972). On the neighborhood effect and the evolution of altruistic traits. Theoretical Population Biology 2: 258-277.

Hamilton, W. D. (1975). Innate social aptitudes in man: An approach from evolutionary genetics. In Fox, R. (ed.), Biosocial Anthropology. Halstead, London, pp. 133-155.

Irons, W. (1979). Natural selection, adaptation, and human social behavior. In Chagnon, N., and Irons, W. (eds.), Evolutionary Biology and Human Social Behavior, Duxbury Press, North Sciutate, Mass.

Kurz, M. (1977). Altruistic equilibrium. In Balassa, B., and Nelson, R. (eds.), Economic Progress, Public Values, and Policy. Elsevier North-Holland, New York, pp. 177-200.

Levin, B., and Kilmer, W. L. (1974). Interdemic selection and the evolution of altruism. Evolution 28: 527-595.

LeVine, R. A., and Campbell, D. T. (1972), Ethnocentrism: Theories of Conflict. Ethnic Attitudes, and Group Behavior. Wiley, New York.

Lumsden, C., and Wilson, E. O. (1981). Genes, Mind, and Culture. Harvard Univ. Press, Cambridge.

Maynard Smith, J. (1976). Group selection. Quarterly Review of Biology 51: 277-283.

Noberini, M. (1966). Ethnocentrism and Feuding: A Cross Cultural Study. MA thesis, Univ. Chicago, Chicago.

Olson, M. (1971). The Logic of Collective Action. Schocken, New York.

Peoples, J. G. (1982). Individual or group advantage? A reinterpretation of the Maring ritual cycle. Current Anthropology 23: 291-310.

Pulliam, H. R., and Dunford, C. (1980). Programmed to Learn. Columbia Univ. Press, New York.

Rappaport, R. A. (1968). Pigs for the Ancestors. Yale Univ. Press, New Haven.

Richerson, P. J., and Boyd, R. (1978). A dual inheritance model of the human evolutionary process. I. Basic postulates and a simple model. Journal of Social and Biological Structures 1: 127-154.

Slatkin, M., and Wade, M. J. (1978). Group selection on a quantitative character. Proceedings of the National Academy of Sciences, U.S.A. 75: 3531-3534.

Shubik, M. (1971). Game theory, behavior, and the paradox of the prisoner's dilemma: Three solutions. Journal of Conflict Resolution. 14: 181-193.

Taylor, M. (1976). Anarchy and Cooperation. Wiley, New York.

Uyenoyama, M. K. (1979). Evolution of altruism under group selection in large and small populations in fluctuating environments. Theoretical Population Biology 15: 58-85.

Uyenoyama, M. K., and Feldman, M. W. (1980). Theories of kin and group selection: A population of genetics perspective. Theoretical Population Biology 17: 380-414.

Van Maanen, J., and Schein, E. H. (1979). Toward a theory of organizational socialization. Research in Organizational Behavior 1: 209-269. 
Wade, M. J. (1978). A critical review of models of group selection. Quarterly Review of Biology 53: 101-114.

Wilson, D. S. (1980). The Natural Selection of Populations and Communities. Benjamin/ Cummings, Reading, Mass. 